\title{
The Effect of Ionic Aggregates on the Transport of Charged Species in Lithium Electrolyte Solutions
}

Giles Richardson ${ }^{1}$, Jamie M. Foster ${ }^{2}$, Athinthra Sethurajan ${ }^{3}$, Sergey

Krachkovskiy $^{4}$, Ion Halalay ${ }^{5}$, Gillian Goward ${ }^{4}$, and Bartosz Protas ${ }^{*}, 6$

${ }^{1}$ Mathematical Sciences, University of Southampton, Highfield, Southampton, United Kingdom SO17 1BJ

${ }^{2}$ Department of Mathematics, University of Portsmouth, Hampshire, United Kingdom PO1 3HF

${ }^{3}$ School of Computational Science and Engineering, McMaster University, Hamilton, ON, Canada L8S 4K1

${ }^{4}$ Department of Chemistry and Chemical Biology, McMaster University, Hamilton, ON, Canada L8S 4K1

${ }^{5}$ General Motors Global R\&D, Warren, Michigan, United States 48090-9055

${ }^{6}$ Department of Mathematics and Statistics, McMaster University, Hamilton, ON, Canada L8S 4K1

June 6, 2018

\begin{abstract}
In this investigation we focus on the problem of modelling the transport of the charged species (lithium ions) in electrolyte solutions with moderate and high salt concentrations $(0.1 \mathrm{M}$ to $>2 \mathrm{M})$, and consider the Nernst-Planck equation as a model of such processes. First, using a combination of magnetic resonance imaging (MRI) and inverse modelling (IM) we demonstrate that at higher concentrations the NernstPlanck equation requires negative transference numbers in order to accurately describe the concentration profiles obtained from experiments. The need for such a physically inconsistent constitutive relation indicates the loss of validity of the Nernst-Planck equation as a model for this process. Next we consider the formation of ion pairs and clusters as a possible effect responsible for the appearance of negative transference numbers and derive an extended version of the Nernst-Planck system which accounts for these additional species. However, a careful analysis of this model reveals that incorporation of ion-pairing effects into the modelling will not change the transference
\end{abstract}

${ }^{*}$ Corresponding Author, Email: bprotas@mcmaster.ca 
numbers inferred from the experimental data via inverse modelling. This demonstrates that physical effects other than formation of ion pairs and clusters must be incorporated into the Nernst-Planck model in order for it to correctly describe ion transport at higher salt concentrations. One prime candidate for such effects is the motion of the reaction surface resulting from dendrite growth.

\section{Introduction}

Modelling plays an ever increasing role in the design of Li-ion batteries which are now used in applications ranging from portable consumer electronics to electrified vehicles. However, modeling efforts are often hampered by the inadequacy of models and the lack, or inaccuracy, of data on the material properties.

A complete and accurate description of the transport of charged species (ions) in electrolyte solutions is an essential component of any model capable of simulating the behavior of actual battery cells. The standard model describing diffusion and migration of ions in dilute electrolyte solutions is the Nernst-Planck (NP) equation [1]. It corresponds to a simplified form of the concentrated-solution theory of mass transport which itself is based on the MaxwellStefan equations. The Nernst-Planck equation, although strictly valid only for very dilute solutions, can be adapted to treat some electrolytes of practical interest by introducing concentration dependence to the mass-transport parameters, i.e. diffusion coefficients $D(c)$ and transference numbers $t^{+}(c)[1,2]$. One way of determining these concentration-dependent material properties is inverse modelling (IM) which is a technique for optimal reconstruction of their form based on suitably matching model predictions to the experimental data. This IM technique has already been applied to determine the material properties of lithium bis(trifluoromethanesulfonyl)imide dissolved in propylene carbonate and the forms of $D(c)$ and $t^{+}(c)$ obtained were shown to be in good agreement with the results obtained using other methods [2]. Since then, we have determined Li concentration profiles in a symmetric Li-Li cell filled with $1 \mathrm{M} \mathrm{LiPF}_{6}$ in a binary mixture of ethylene carbonate (EC) and dimethyl carbonate (DMC) with 1:1 ratio by volume under galvanostatic conditions. Applying the IM technique to the data obtained from this experiment leads to negative values of the transference number $t^{+}(c)<0$ at large concentrations. For binary electrolytes based on lithium salts and neutral organic carbonates, such as the one used in our study, negative transference numbers are not possible $[3,4]$. However, negative values of lithium transference numbers can occur for lithium salt/ionic liquid ternary mixtures with two cations and common anion, as reported in [5]. It has long been known that ion aggregation occurs in certain electrolytes, as has been deduced by Onsager from experiments in which the conductivity increases with field strength $[6,7]$. Initially we attributed the predicted negative transference numbers to the omission of ionic aggregation from the model noting that: (i) there is good evidence that such species can form in $\mathrm{LiPF}_{6}$ in $\mathrm{EC}$ :DMC which has a relatively low dielectric permittivity $(\varepsilon \approx 30)$ and, therefore, exhibits a high degree of ion association (ion pairing $>50 \%$ ) [3], (ii) NMR experiments detect all Li nuclei independently of whether they are in the form of free cations or part of a larger aggregate, and (iii) ion pairs, being neutral, do not perform a migration motion in an applied electric field. Moreover, it has been postulated that a phenomenon which could give rise to negative transference numbers in binary systems is the 
formation and transport of ionic aggregates which form and disintegrate spontaneously at increased electrolyte concentrations [8]. We demonstrate in the present study that incorporation of ionic aggregation into an extended Nernst-Planck model does not in fact change the transference numbers that can be inferred from the data. It is therefore apparent that in order to obtain an accurate model of ion transport at high concentrations, one must augment the Nernst-Planck model with other physical effects.

The structure of the paper is as follows. In Section 2 we describe the experiment used to acquire the data. In Section 3 the standard Nernst-Planck model is introduced, together with all modelling assumptions, and we present results indicating the loss of its validity at moderate and high salt concentrations. An augmented model accounting for the possibility that ion pairs can form and disintegrate is introduced in Section 4, where careful analysis demonstrates that in the limit of fast reaction rates this augmented model remains formally equivalent to the original Nernst-Planck system. A discussion and final conclusions are provided in Section 5 whilst additional details, including a further extension to higher order aggregates with the same conclusion is presented in the appendices.

\section{Experimental}

The experiment monitors the gradual build-up of the ionic concentration gradient in an electrolyte solution which results from the application of a constant current, starting from an initially uniform concentration throughout the solution volume. The experiment is carried out under galvanostatic conditions in a symmetric Li-Li electrochemical cell constructed from a $5 \mathrm{~mm}$ diameter NMR tube, shown in Figure 1, filled with a $1 \mathrm{M} \mathrm{LiPF} 6$ solution in a binary mixture of ethylene carbonate (EC) and dimethyl carbonate (DMC) with 1:1 volume ratios. A constant current of $75 \mu \mathrm{A}$ (corresponding to the current density of 382 $\mu \mathrm{A} \mathrm{cm} \mathrm{cm}^{-2}$ ) was applied to the cell for 16 hours. Concentration profiles were acquired using magnetic resonance imaging (MRI). For this experiment we chose to monitor the ${ }^{19} \mathrm{~F}$ nuclei, which significantly reduces the data acquisition time, since the relative NMR sensitivity to a ${ }^{19} \mathrm{~F}$ nucleus is approximately 3 times higher than to a ${ }^{7} \mathrm{Li}$ nucleus. One-dimensional ${ }^{19} \mathrm{~F}$ NMR images were obtained using a gradient spin-echo pulse sequence with the magnetic field gradient applied along the $x$-direction (i.e., along the axis of the cell), with a $3 \mathrm{~ms}$ echo time and a $20 \mathrm{G} / \mathrm{cm}$ reading gradient [9]. In the course of the experiment 256 frequencydomain points were collected over the spectral width of $200 \mathrm{kHz}$. The combination of the magnetic field gradient and spectral resolution yielded a spatial resolution of $40 \mu \mathrm{m}$. A total of 64 scans with a relaxation delay of $3.5 \mathrm{~s}$ were collected for each image, resulting in an acquisition time of 4 minutes per image. The imaging measurement sequence was repeated at 2-hour intervals uniformly spread over 14 hours duration of the galvanostatic experiment. The experimentally obtained concentration profiles, hereafter denoted $\tilde{c}(x, t)$, are shown in Figure 1 at different times $t \in[0,14$ hours $]$ as functions of the space coordinate $x$.

\section{The Nernst-Planck Model}

In the present section we recall the classical Nernst-Planck model used to describe the transport of charged species in dilute electrolytes [1]. The concentrations of cations and anions 
are denoted by $c_{+}$and $c_{-}$, respectively. We make the following modelling assumptions in order to obtain the mathematical description of the mass transport during the galvanostatic experiment described in Section 2:

A1: isothermal conditions;

A2: the driving force for mass transport of a species is the gradient of its chemical potential;

A3: the lack of thermodynamic ideality (i.e., activity coefficient different from one) and the effect of the solution viscosity are accounted for by an a priori undetermined dependence of the material properties on the salt concentration;

A4: ion transport occurs only in the axial direction and transport in the radial direction of the cell is negligible;

A5: the electrolyte solution is homogeneous at the beginning of the experiment;

A6: the system satisfies local electrical neutrality at every location in the bulk, which implies that $c_{+}=c_{-}=c$, were $\mathrm{c}$ is the salt concentration;

A7: mass transport occurs only by diffusion and migration in the applied electric field (i.e., convective transport is neglected);

A8: the cation flux at the two boundaries $(x=0$ and $x=L)$ corresponds to the applied electric current and results in lithium deposition and stripping, respectively $[10,1]$.

We therefore consider a $1 \mathrm{D}$ problem with the spatial coordinate $x \in[0, L]$, where $L$ is the length of the electrolyte-filled region in the cell, and time $t \in[0, T]$, where $T$ denotes the duration of the experiment. The above assumptions lead to the following partial differential equation (PDE) describing mass transport in the electrolyte solution (1a), subject to the boundary conditions (1b) and the initial condition (1c):

$$
\begin{aligned}
\frac{\partial c}{\partial t} & =\frac{\partial}{\partial x}\left[D \frac{\partial c}{\partial x}+\frac{\left(1-t^{+}\right) I}{F A}\right] & & \text { in }(0, L) \times(0, T], \\
\left.\frac{\partial c}{\partial x}\right|_{x=0, L} & =-\frac{\left(1-t^{+}\right) I}{D F A} & & \text { in }(0, T], \\
\left.c\right|_{t=0} & =c^{\text {init }} & & \text { in }(0, L),
\end{aligned}
$$

where $c^{\text {init }}$ is the initial concentration, $A$ is the cross-sectional area of the cell, $F$ is Faraday's constant, whereas $I$ denotes the applied constant current. We note that the effective Fickian diffusion coefficient $D$ and the transference number $t^{+}$are considered unknown and will be reconstructed from the experimental data using the inverse modelling approach described in the following subsection. In contrast to the standard Nernst-Planck theory, both the diffusion coefficient and the transference number will be considered functions of the concentration $c$ in our model, i.e., $D=D(c)$ and $t^{+}=t^{+}(c)$. 


\subsection{Material Properties Estimates Based on the Nernst-Planck Model}

In our prior investigation [2] the inverse modelling approach (as briefly described in Appendix A) was applied to infer material properties of lithium bis(trifluoromethanesulfonyl)imide solutions in propylene carbonate with concentrations up to $1.1 \mathrm{M}$ and produced thermodynamically consistent reconstructions of the effective diffusivity and $\mathrm{Li}^{+}$transference number as functions of the salt concentration. However, when this approach is applied to the concentration profiles shown in Section 2, cf. Figure 1, which feature salt concentrations up to 2.2 $\mathrm{M}$, the results shown in Figure 1 are no longer physically consistent. Specifically, while the reconstruction of the diffusion coefficient $D(c)$ is in the expected range, the reconstructed values of the $\mathrm{Li}^{+}$transference number $t^{+}(c)$ are negative for most of salt concentrations, i.e., at $>0.85 \mathrm{M}$. Under assumptions A1-A8, a negative transference number $t^{+}$implies that the cationic current due to migration has a direction opposite to the electric field which is physically impossible for binary symmetric electrolytes. At the same time, we emphasize that the negative transference numbers were obtained as the constitutive relation in system (1) that allows this system to optimally match the experimental data, in the sense of minimization of the least-square error (for details, see the minimization problems P1 and P2 defined in Appendix A). Therefore, such a result demonstrates that system (1) no longer provides a physically valid description of the data. This failure is clearly attributable to the fact that system (1) does not account for physical effects which become important at higher concentrations. Such effects may include the formation of neutral ion pairs, different forms of advection [11] or the motion of the reaction surface as dendrites are formed on the electrode surfaces. We will discuss the former effect in the next section.

\subsection{Prior Reports of Negative Cation Transference Numbers}

There exist several prior studies that report negative transference numbers and speculate about their possible origins. For example, investigation [12] provides evidence for a negative transference number measured in a lithium binary symmetric electrolyte. While a number of possible reasons for negative transference numbers is mentioned by the authors, they conclude that the formation of large complexes and a high molecular weight of the anion may be the key factor. An analogous opinion is expressed in monograph [13] where the authors argue that formation of ion complexes of cations and anions with a net negative charge at higher salt concentration in a non-symmetric electrolyte can result in a negative transference number. This hypothesis was also reinforced by other studies [14]. The thermodynamic validity of negative transference number was considered in [15]. Based on the analysis presented in that study, one can conclude that in principle negative transference numbers may arise under certain conditions in non-binary electrolytes. Since the experimental set-up discussed in Section 2 involves a binary electrolyte, this argument cannot explain the results reported in Section 3.1, cf. Figure 2. Hence, in the next section we will address the question whether ion-paring effects could be used to justify negative transference numbers. 


\section{Transport Model with Ion-Pairing Effects}

In this section we formulate a generalized version of the Nernst-Planck model discussed in Section 3, in which the effects of ion-pairing are explicitly accounted for. We will then draw some conclusions about how this extension affects the reconstructions of material properties via inverse modelling. Henceforth, we will use the term "ion pair" to describe complexes that are formed when a positive and negative ion come sufficiently close together to become (reversibly) bound by their Coulombic interaction. The formation of an ion pair could then occur via the reaction $\mathrm{Li}^{+}+A^{-} \rightleftharpoons \mathrm{LiA}$, where $A^{-}$represents the anion in the binary symmetric salt $L i A$. Of course, it is conceivable that higher-order clusters (consisting of more than one of either the positive and negative ions) may form in electrolytic solutions and in the recent literature there exist reports based on molecular dynamics (MD) simulations indicating the presence of higher-order clusters in Li-ion battery electrolytes [16]. We note, however, that MD probes phenomena on time scales several orders of magnitude faster than the time scales for diffusion in liquids and no reports regarding the lifetime of higher-order clusters were provided. Furthermore, the absence of the signature typical for the existence of substantial amounts of triple ions in the ionic conductivity data for $\mathrm{LiPF}_{6} /$ carbonates solutions is also reported [17]. Therefore, for simplicity, in this section we neglect the presence of higher-order ion clusters. However, this analysis arrives at the same conclusions when extended to higher-order clusters, as demonstrated in Appendix D.

Conservation equations for each of the three species in the system can be written as follows

$$
\begin{aligned}
\frac{\partial c_{+}}{\partial t}+\frac{\partial \mathcal{F}_{+}}{\partial x} & =-Q\left(c_{+}, c_{-}, c_{o}\right), \\
\frac{\partial c_{-}}{\partial t}+\frac{\partial \mathcal{F}_{-}}{\partial x} & =-Q\left(c_{+}, c_{-}, c_{o}\right), \\
\frac{\partial c_{o}}{\partial t}+\frac{\partial \mathcal{F}_{o}}{\partial x} & =Q\left(c_{+}, c_{-}, c_{o}\right),
\end{aligned}
$$

where $c_{+}, c_{-}$, and $c_{o}$, are the cation, anion and ion-pair concentrations, respectively, $\mathcal{F}_{+}$, $\mathcal{F}_{-}$, and $\mathcal{F}_{o}$, are the corresponding fluxes (specified in Appendix B), whereas $Q\left(c_{+}, c_{-}, c_{o}\right)$ is the rate of the ion-pair formation reaction. In symmetrical electrochemical cells with lithium metal electrodes, lithium is stripped from one electrode deposited onto the other. The possible electrochemical reactions that can lead to lithium deposition/stripping are [18]

$$
\begin{aligned}
& \text { (I) } \quad L i \rightleftharpoons L i^{+}+e^{-}, \\
& \text {(II) } L i+A^{-} \rightleftharpoons L i A+e^{-} .
\end{aligned}
$$

For reaction (I) we denote the forward reaction rate (per unit area) by $r_{(\mathrm{I})}$, on the electrode at $x=0$, and by $R_{(\mathrm{I})}$, on the electrode at $x=L$. For reaction (II) we denote the forward reaction rate (per unit area) by $r_{(\mathrm{II})}$ on $x=0$ and by $R_{(\mathrm{II})}$ on $x=L$. The appropriate 
boundary conditions on the ionic fluxes are thus

$$
\begin{aligned}
\left.\mathcal{F}_{+}\right|_{x=0} & =r_{(\mathrm{I})}, \\
\left.\mathcal{F}_{-}\right|_{x=0} & =-r_{(\mathrm{II})}, \\
\left.\mathcal{F}_{o}\right|_{x=0} & =r_{(\mathrm{II})} . \\
\left.\mathcal{F}_{+}\right|_{x=L} & =-R_{(\mathrm{I})}, \\
\left.\mathcal{F}_{-}\right|_{x=L} & =R_{(\mathrm{II})}, \\
\left.\mathcal{F}_{o}\right|_{x=L} & =-R_{(\mathrm{II})} .
\end{aligned}
$$

which can, in turn, be related to the current density flowing through the device via

$$
\left.j\right|_{x=0}=F\left(r_{(I)}+r_{(I I)}\right), \quad \text { and }\left.\quad j\right|_{x=L}=-F\left(R_{(I)}+R_{(I I)}\right) .
$$

Charge neutrality. As is usual in such charge transport problems, at realistic ion concentrations there is almost exact charge neutrality

$$
c_{-} \approx c_{+}
$$

throughout nearly all of the electrolyte, except in very narrow double layers adjacent to the electrodes (typically of size around $1 \mathrm{~nm}$ ). This is a consequence of Poisson's equations and the very short Debye length of the electrolytes used in battery applications.

Reaction quasi-equilibrium. Borodin et al. [19] estimate the reaction rate for the dimerisation reaction $k$ to be of the order of $10^{9} \mathrm{~s}^{-1}$ and this allows us to determine how close to quasi-equilibrium the dimerisation reaction will be. In the immediate vicinity of the electrodes we do not expect the reaction to be close to equilibrium because the reactions occurring there act to drive the system away from equilibrium. However, by comparing the timescale for diffusion of ions $L^{2} / D$ to the reaction rate timescale $1 / k$ we can obtain an estimate of the length scale $L$ away from the electrodes over which the system shows significant deviation from equilibrium. With an estimate of $D \sim 10^{-9} \mathrm{~m}^{2} \mathrm{~s}^{-1}$ this gives $L \approx 1 \mathrm{~nm}$. Thus we expect the dimerisation reaction to be at quasi-equilibrium throughout the electrolyte except in narrow reaction layers, of width $\approx 1 \mathrm{~nm}$, in the immediate vicinity of the electrodes.

The bulk equations. Away from the Debye layers and reaction layers lying adjacent to the electrodes we expect almost exact charge neutrality and almost exact equilibrium of the dimerisation reaction. These two assumptions allow us to considerably simplify the governing equations by writing

$$
c_{-}=c \quad \text { and } \quad c_{+}=c, \quad \text { (charge neutrality) }
$$

and

$$
c_{0}=F(c), \quad \text { (quasi-equilibrium of dimerisation reaction). }
$$

Here the equilibrium function $F(c)$ can be obtained by balancing the chemical potential of the electrolyte with that of the dimer (see equation (25) in Appendix B). We note that a 
mass action balance, as considered for example in [20], would give $\left[c_{+}\right]\left[c_{-}\right]=M_{F}\left[c_{0}\right]^{2}$, with an equilibrium constant $M_{F}$. Therefore, we would have $F(c)=M_{F} c^{2}$ in (7), which is a special case of the present more general approach. In (6) and (7) we therefore have two equations for the three variables $c_{+}, c_{-}$and $c_{0}$, and we need a further equation and appropriate boundary conditions that will allow us to fully determine these variables.

A transport equation for total Lithium concentration in the bulk. In order to close the problem for $c_{+}, c_{-}$and $c_{0}$ in the bulk region (away from the Debye and reaction layers adjacent to the electrodes) we seek a diffusion equation for the total lithium concentration in the electrolyte

$$
[\mathrm{Li}]=\left[\mathrm{Li}^{+}\right]+[\mathrm{LiA}]=c_{+}+c_{o} .
$$

This should be in a form in which the dimerisation reaction rate $Q$ does not appear explicitly. Because although the reaction lies close to quasi-equilibrium it is not necessarily true that the $Q$ terms in (2a)-(2c) are negligible. In addition, given the experiment that we are trying to model, we require a relation between the total lithium flux and the current density at the electrodes. Throughout nearly all the electrolyte (with the exception of the Double layers) charge neutrality $c_{-}=c_{+}$is satisfied and we can rewrite $(8)$ in the form $[\mathrm{Li}]=\frac{1}{2}\left(c_{+}+c_{-}\right)+c_{o}$. This motivates us to define the total lithium concentration $c_{T}$ and the total lithium flux $\mathcal{F}_{T}$ (outside the double layers) by the expressions

$$
c_{T}=\frac{1}{2}\left(c_{+}+c_{-}\right)+c_{0}, \quad \text { and } \quad \mathcal{F}_{T}=\frac{\mathcal{F}_{-}+\mathcal{F}_{+}}{2}+\mathcal{F}_{0}
$$

We note that while $c_{T}$ does not represent the total lithium concentration [Li] in the narrow double layers, it is still possible to write down a conservation equation for $c_{T}$ throughout the entire electrolyte (including the double layers). This is accomplished by adding twice (2c) to (2a) and (2b) and dividing the result by two which yields an equation that is independent of the volumetric reaction rate $Q$, namely

$$
\frac{\partial c_{T}}{\partial t}+\frac{\partial \mathcal{F}_{T}}{\partial x}=0
$$

Appropriate boundary conditions on this conservation equation for $c_{T}$ can be derived by re-expressing $\mathcal{F}_{T}$ on the boundaries in terms of the reaction rates there, via (4a)-(4f) and (5). This results in two conditions that are independent of the reactions occurring in the boundary layers

$$
\left.\mathcal{F}_{T}\right|_{x=0}=\frac{j(t)}{2 F}, \quad \text { and }\left.\quad \mathcal{F}_{T}\right|_{x=L}=\frac{j(t)}{2 F}
$$

The total lithium flux $\mathcal{F}_{T}$ is related to total lithium concentration $c_{T}$, except in the narrow double and reaction layers adjacent to the electrodes, via the relation

$$
\mathcal{F}_{T}=-\widetilde{D}\left(c_{T}\right) \frac{\partial c_{T}}{\partial x}-\frac{j}{2 F}\left(1-2 t^{+}\right) .
$$

which is derived in Appendix B, cf. equation (33). Even though this definition of the flux does not apply in the double and reaction layers, it holds in the central bulk region that 
extends across nearly the entire electrolyte. Since double and reaction layers are narrow, their capacity for ions and ion pairs is low and consequently the total flux $\mathcal{F}_{T}$ across them is almost uniform (an argument that has been formalized by conducting a boundary layer analysis of equation (10), see appendix C). Thus the boundary conditions (11) can be applied directly to the bulk flux $\mathcal{F}_{T}$, as defined in (12), even though this relation does not hold in the double and reaction layers adjacent to the reaction surfaces $x=0, L$.

We note that when we substitute equation (12) for the flux into the conservation equation (10) and the boundary conditions (11), we retrieve equations (1a)-(1b) which were used in the original inverse modelling approach, namely,

$$
\begin{array}{r}
\frac{\partial c_{T}}{\partial t}=\frac{\partial}{\partial x}\left(\widetilde{D}\left(c_{T}\right) \frac{\partial c_{T}}{\partial x}\right)-\frac{\partial t^{+}}{\partial x} \frac{j}{F} \\
\left.\widetilde{D}\left(c_{T}\right) \frac{\partial c_{T}}{\partial x}\right|_{x=0}=\frac{j}{F}\left(1-t^{+}\right) \\
\left.\widetilde{D}\left(c_{T}\right) \frac{\partial c_{T}}{\partial x}\right|_{x=L}=\frac{j}{F}\left(1-t^{+}\right) .
\end{array}
$$

where here $j$ is constant and related to the total current $I$ via $j=I / A$. It is thus immediately apparent that invoking ion pairing cannot resolve the issue of negative transference numbers, since the system for the total lithium concentration that we obtain here is identical to the original system investigated in the inverse-modelling approach used to determine the electrolyte properties.

\section{Conclusions and Outlook}

The equivalence of the reduced system (13)-(15) with the effective diffusion coefficient $\widetilde{D}$, cf. (32), and the original Nernst-Planck model (1) demonstrates that accounting for ionpairing under the assumption of fast reaction rates cannot affect the transference numbers reconstructed via inverse modelling. In particular, incorporation of this effect will not resolve the problem highlighted in Section 3.1 where negative transference numbers were obtained from reconstructions. As demonstrated in Appendix D, this conclusion still holds even when higher-order ion clusters are taken into account. These findings therefore call into question the claim made in [14] about the relation between negative transference numbers and ion pairing. Since the extended model introduced in Section 4 which accounts for ion pairing still cannot provide a physically consistent description of the galvanostatic experiment (cf. Section 2 ), one needs to consider other physical effects not accounted for in the Nernst-Planck model. One such candidate is incorporation of the motion of the reaction surface owing to dendrite growth, and inverse modelling in the presence of such effects will be the topic in a forthcoming report. 


\section{Appendices}

\section{A Inverse Modelling}

The unknown material properties, $D$ and $t^{+}$, can be reconstructed based on the assumed transport model, such as system (1), and using the concentration profiles obtained in the NMR experiment described in Section 2. We will use the inverse modelling approach developed and validated in [2] in which the problem is framed as minimization of a cost functional representing the least-squares deviation between the concentration values predicted by the model (denoted $c$ in eq. (1)) and the experimentally determined concentration values $\tilde{c}$. The cost functional can thus be represented as

$$
\mathcal{J}\left(D, t^{+}\right)=\frac{1}{2} \int_{0}^{T} \int_{0}^{L}\left[c\left(x, t ; D, t^{+}\right)-\tilde{c}(x, t)\right]^{2} d x d t
$$

We will consider two distinct formulations corresponding, respectively, to constant and to concentration-dependent material properties. The first one pertains to the standard NernstPlanck theory, whereas the second one represents the extension discussed in [2]. We will henceforth distinguish the two cases by using the following notations: $D$ and $t^{+}$for the former case, $D(c)$ and $t^{+}(c)$ for the latter case.

When both $D$ and $t^{+}$are constant, we obtain a simple optimization problem (which is exact in the limiting case of an ideal solution, i.e., at very dilute salt concentrations)

$$
\mathrm{P} 1: \quad\left[\widehat{D}, \widehat{t}^{+}\right]=\underset{\left[t^{+}, D\right] \in \mathbb{R}^{2}}{\operatorname{argmin}} \mathcal{J}\left(D, t^{+}\right)
$$

(henceforth carets ". " will denote optimal reconstructions). Problem P1 is rather well understood and can be solved in a straightforward manner using commercially available software tools such as the minimization routines in MATLAB. It was in fact already solved in the seminal study by Klett et al. [21] and is also solved here as a preliminary step in a more complete analysis.

A more complicated optimization problem arises when both $D(c)$ and $t^{+}(c)$ are concentrationdependent, which reflects the physics of the problem in more realistic fashion

$$
\mathrm{P} 2: \quad\left[\widehat{D}(c), \widehat{t}^{+}(c)\right]=\underset{\left[t^{+}(c), D(c)\right] \in \mathcal{X}}{\operatorname{argmin}} \mathcal{J}\left(D(c), t^{+}(c)\right),
$$

where $\mathcal{X}$ denotes a suitable function space to which $D(c)$ and $t^{+}(c)$ belong. We emphasize that, apart from smoothness and the limiting behavior for large and small values of $c$, no other a priori assumptions are made about the functional forms of $D(c)$ and $t^{+}(c)$. In contrast to the simplified problem $\mathrm{P} 1$, the computational approach required to solve the more realistic problem $\mathrm{P} 2$ with concentration-dependent material properties is more involved and necessitates specialized tools described in detail in $[22,23]$.

\section{B The Total Lithium Flux in the Bulk}

In this appendix we give the details behind the modification to the Nernst-Planck equations accounting for ion pairing. We begin by stating expressions for the fluxes of positive ions 
$\mathcal{F}_{+}$, negative ions $\mathcal{F}_{-}$and ion pairs $\mathcal{F}_{o}$ using the Maxwell-Stefan diffusion formalism which is applicable to moderately concentrated electrolytes [15]

$$
\begin{aligned}
\mathcal{F}_{+}=-\frac{D_{+} c_{+}}{R T} \frac{\partial \mu_{+}}{\partial x}, & \mu_{+}=\mu_{+}^{\ominus}+R T \log a_{+}+F \phi, \\
\mathcal{F}_{-}=-\frac{D_{-} c_{-}}{R T} \frac{\partial \mu_{-}}{\partial x}, & \mu_{-}=\mu_{-}^{\ominus}+R T \log a_{-}-F \phi, \\
\mathcal{F}_{o}=-\frac{D_{o} c_{o}}{R T} \frac{\partial \mu_{o}}{\partial x}, & \mu_{o}=\mu_{o}^{\ominus}+R T \log a_{o},
\end{aligned}
$$

where $D_{k}, \mu_{k}^{\ominus}$ and $\mu_{k}$, and $a_{k}$ are, respectively, the diffusion coefficient, the reference and total electrochemical potential and activity of the $k$ th species (for $k=+,-, o$ ), whereas $\phi$ is the electric potential. Within the electrolyte and away from the double layers at the electrode there is almost exact charge electroneutrality, i.e. $c_{+}=c_{-}$. This allows us to write

$$
c_{+}=c, \quad \text { and } \quad c_{-}=c .
$$

Substituting these electroneutrality relations into equations (2a)-(2b) and subtracting these two equations gives the following relation for current conservation

$$
\frac{\partial j}{\partial x}=0, \quad \text { where } \quad j=F\left(\mathcal{F}_{+}-\mathcal{F}_{-}\right),
$$

in which $j$ is the current density. On substituting for $\mathcal{F}_{+}$and $\mathcal{F}_{-}$from (17a)-(17b), the expression for $j$ may also be written as

$$
j=-\frac{F c}{R T}\left(D_{+} \frac{\partial \mu_{+}}{\partial x}-D_{-} \frac{\partial \mu_{-}}{\partial x}\right) .
$$

At this stage it is convenient to introduce the electrolyte chemical potential $\mu_{e}$ defined as

$$
\mu_{e}=\frac{\mu_{+}+\mu_{-}}{2}
$$

which has the property that it is independent of the electric potential, since by using the definitions of the electrochemical potentials found in (17a)-(17b) and (21) we see that

$$
\mu_{e}=\mu_{e}^{\ominus}+R T \log \left(a_{e}(c)\right), \quad \text { where } \quad a_{e}(c)=\left[a_{+}(c) a_{-}(c)\right]^{1 / 2} \quad \text { and } \quad \mu_{e}^{\ominus}=\frac{\mu_{+}^{\ominus}+\mu_{-}^{\ominus}}{2}
$$

Where the dimerisation reaction $\mathrm{Li}^{+}+\mathrm{A}^{-} \leftrightharpoons \mathrm{LiA}$ is in quasi-equilibrium, the following relation between the chemical potentials is satisfied

$$
\mu_{+}+\mu_{-}=\mu_{o}
$$

which in turn implies that

$$
a_{o}=K a_{e}^{2}, \quad \text { where } \quad K=\exp \left(\frac{2 \mu_{e}^{\ominus}-\mu_{o}^{\ominus}}{R T}\right),
$$


and since $a_{e}=a_{e}(c)$ and $a_{o}=a_{o}\left(c_{o}\right)$, this implies a functional relationship between $c_{o}$ and $c$. Since we will be working with the "total concentration" $c_{T}=c_{o}+c$, as defined in (9), it is most helpful to express this in the functional form

$$
c_{o}=f\left(c_{T}\right)
$$

from which it follows that

$$
c=c_{T}-f\left(c_{T}\right)
$$

We now seek to express the ion fluxes, as defined in (17a)-(17b), solely in terms of the electrolyte chemical potential $\mu_{e}$ and the current density $j$. We do this (following [1]) by referring to relation (20) and recasting (17a)-(17b) in the following form

$$
\begin{aligned}
& \mathcal{F}_{+}=-\frac{c}{R T}\left[D_{+} \frac{\partial \mu_{+}}{\partial x}-\alpha\left(D_{+} \frac{\partial \mu_{+}}{\partial x}-D_{-} \frac{\partial \mu_{-}}{\partial x}\right)\right]+\alpha \frac{j}{F} \\
& \mathcal{F}_{-}=-\frac{c}{R T}\left[D_{-} \frac{\partial \mu_{-}}{\partial x}-\beta\left(D_{+} \frac{\partial \mu_{+}}{\partial x}-D_{-} \frac{\partial \mu_{-}}{\partial x}\right)\right]+\beta \frac{j}{F}
\end{aligned}
$$

with an appropriate choice of $\alpha$ and $\beta$. In this instance, by taking $\alpha=t^{+}$and $\beta=-\left(1-t^{+}\right)$, where the transference number $t^{+}$is defined via

$$
t^{+}=\frac{D_{+}}{D_{-}+D_{+}}
$$

we obtain the desired expressions, namely,

$$
\begin{aligned}
& \mathcal{F}_{+}=-\frac{c}{R T} \frac{2 D_{+} D_{-}}{D_{+}+D_{-}} \frac{\partial \mu_{e}}{\partial x}+\frac{t^{+} j}{F} \\
& \mathcal{F}_{-}=-\frac{c}{R T} \frac{2 D_{+} D_{-}}{D_{+}+D_{-}} \frac{\partial \mu_{e}}{\partial x}-\frac{\left(1-t^{+}\right) j}{F} .
\end{aligned}
$$

Defining

$$
\mathcal{D}^{\dagger}(c)=c \frac{2 D_{+} D_{-}}{D_{+}+D_{-}} \frac{\partial \mu_{e}}{\partial a_{e}} \frac{\partial a_{e}}{\partial c}=c \frac{2 D_{+} D_{-}}{D_{+}+D_{-}} \frac{R T}{a_{e}} \frac{\partial a_{e}}{\partial c}
$$

where we used relation (22), we can rewrite the fluxes as

$$
\begin{aligned}
& \mathcal{F}_{+}=-\mathcal{D}^{\dagger}(c) \frac{\partial c}{\partial x}+\frac{t^{+} j}{F} \\
& \mathcal{F}_{-}=-\mathcal{D}^{\dagger}(c) \frac{\partial c}{\partial x}-\frac{\left(1-t^{+}\right) j}{F}
\end{aligned}
$$

Similarly, defining $\mathcal{D}_{o}^{\dagger}\left(c_{o}\right)=c_{o} D_{o} \frac{\partial \mu_{o}}{\partial a_{o}} \frac{\partial a_{o}}{\partial c_{o}}$, we have

$$
\mathcal{F}_{o}=-\mathcal{D}_{o}^{\dagger}\left(c_{o}\right) \frac{\partial c_{o}}{\partial x}
$$


Then, using the definition of the total lithium flux $\mathcal{F}_{T}$ found in (9) together with the relations (29a)-(29b) and (30), we obtain the following expression

$$
\mathcal{F}_{T}=-\left(\mathcal{D}^{\dagger}(c) \frac{\partial c_{T}}{\partial x}\left(1-\frac{d f}{d c_{T}}\right)+\mathcal{D}_{o}^{\dagger}\left(c_{o}\right) \frac{d f}{d c_{T}}\right) \frac{\partial c_{T}}{\partial x}-\frac{j}{2 F}\left(1-2 t^{+}\right),
$$

where $c_{o}$ and $c$ are given by (25) and (26), respectively. It is straightforward to rewrite this expression in terms of an effective diffusivity, defined by

$$
\widetilde{D}\left(c_{T}\right)=\mathcal{D}^{\dagger}(c)+\left(\mathcal{D}_{o}^{\dagger}\left(c_{o}\right)-\mathcal{D}^{\dagger}(c)\right) \frac{d f\left(c_{T}\right)}{d c_{T}},
$$

so that it now reads

$$
\mathcal{F}_{T}=-\widetilde{D}\left(c_{T}\right) \frac{\partial c_{T}}{\partial x}-\frac{j}{2 F}\left(1-2 t^{+}\right)
$$

\section{Fluxes in the Reaction Layers}

In this section we offer a systematic demonstration that the fluxes of the charged species do not vary appreciably across the narrow reaction layers in the vicinity of the interfaces using asymptotic methods. First, we cast the problem in nondimensional form by scaling the dependent variables. Henceforth, quantities marked with a star are dimensionless. We write

$$
\begin{array}{lll}
c_{i}=\hat{c} c_{i}^{*}, & Q=k \hat{c} Q^{*}, & x=L x^{*}, \\
\mathcal{F}_{i}=\frac{L \hat{c}}{\tau} \mathcal{F}_{i}^{*}, & R_{i}=\frac{L \hat{c}}{\tau} R_{i}^{*}, & t=\tau t^{*},
\end{array}
$$

where $\hat{c}$ is a typical value of the ionic concentration, $\mathcal{F}_{i}$ is the flux of species $i=\{+,-, o\}$, $\tau$ is a characteristic timescale for the experiment, whereas $k$ is a characteristic reaction rate. Typical values of some of these parameters are summarized in Table 1. On applying these scaling relations to equations (2) as well as (10) and its boundary conditions (11) we obtain

$$
\begin{array}{r}
\frac{\partial c_{+}^{*}}{\partial t^{*}}+\frac{\partial \mathcal{F}_{+}^{*}}{\partial x^{*}}=-\frac{Q^{*}}{\delta}, \quad \frac{\partial c_{-}^{*}}{\partial t^{*}}+\frac{\partial \mathcal{F}_{-}^{*}}{\partial x^{*}}=-\frac{Q^{*}}{\delta}, \quad \frac{\partial c_{o}^{*}}{\partial t^{*}}+\frac{\partial \mathcal{F}_{o}^{*}}{\partial x^{*}}=\frac{Q^{*}}{\delta} \\
\frac{\partial c_{T}^{*}}{\partial t^{*}}+\frac{\partial \mathcal{F}_{T}^{*}}{\partial x^{*}}=0,\left.\quad \mathcal{F}_{T}^{*}\right|_{x=0}=\frac{J}{2},\left.\quad \mathcal{F}_{T}^{*}\right|_{x=1}=\frac{J}{2}
\end{array}
$$

where

$$
\delta=\frac{1}{\tau k} \ll 1, \quad J=\frac{j}{F \mathcal{F}_{i}^{*}}=O(1) .
$$

Here $\delta$ is the ratio of the typical timescale of experiment to those for ion-pairing reactions while $J$ is the ratio of the electronic current density supplied at the contact to the ionic current density in the solution.

The small value of $\delta$ (which can be estimated using the parameter values in Table 1 ) requires that the bulk of the electrolyte, where $x^{*}=O(1)$, be in quasi-equilibrium. Contrastingly, 
close to the edges of the domain near the electrodes, where either $x^{*}=O(\delta)$ or $1-x^{*}=O(\delta)$, the reaction rate could be sufficiently large that it may appreciably alter the fluxes of some of the different species across these narrow layers. Examining (35a)-(35b) we see that the fluxes of anions, cations and neutral ion pairs are all altered, but, crucially, the flux of the total mount of Lithium is unaffected. To demonstrate this we make the following rescalings in order to form the governing equations in the narrow (of width $O(\delta)$ ) reaction layers

$$
x^{*}=\delta w_{l}^{*}, \quad 1-x^{*}=\delta w_{r}^{*},
$$

so that $w_{l}$ and $w_{r}$ are the local coordinates within the left- and right-hand non-equilibrium layers, respectively. Under these rescalings the governing system for $c_{T}^{*}$, cf. relation (35b), becomes

$$
\frac{\partial c_{T}^{*}}{\partial t^{*}}+\frac{1}{\delta} \frac{\partial \mathcal{F}_{T}^{*}}{\partial w_{l, r}^{*}}=0
$$

which, on noting the smallness of $\delta$, immediately asserts that

$$
\mathcal{F}_{T}^{*}=\frac{j}{2 F}+\mathcal{O}(\delta)
$$

so one can thus write

$$
\left.\mathcal{F}_{T}^{*}\right|_{x^{*}=0^{+}, 1^{-}}=\frac{j}{2 F}+\mathcal{O}(\delta)
$$

throughout the reaction layers adjacent to the electrodes. This more rigorously justifies the application of the boundary conditions (11) to the governing system for the total Lithium flux in the bulk (10).

\section{Higher-Order Ion Clusters}

Here we extend the analysis of Section 4 to demonstrate that in fact the same conclusions also hold when higher-order ion complexes are taken into account. Let us now consider third-order ion clusters with concentrations denoted as

$$
\begin{aligned}
& c_{1+} \text { - concentration of } \mathrm{Li}^{+}, \\
& c_{1-} \text { - concentration of } \mathrm{N}^{-}, \\
& c_{20} \text { - concentration of } \mathrm{LiN}, \\
& c_{3+} \text { - concentration of } \mathrm{Li}_{2} \mathrm{~N}^{+}, \\
& c_{3-}-\text { concentration of } \mathrm{LiN}_{2}^{-} .
\end{aligned}
$$

Assuming that third-order ion clusters form from ion pairs, we can write the transformation reactions as follows

$$
\begin{aligned}
Q_{1}: & \quad L i^{+}+N^{-} \rightleftharpoons \operatorname{LiN}, \\
Q_{2}: & L i N+L i^{+} \rightleftharpoons L_{2} N^{+}, \\
Q_{3}: & L i N+N^{-} \rightleftharpoons L_{i} N_{2}^{-},
\end{aligned}
$$


where $Q_{1}, Q_{2}$ and $Q_{3}$ are the corresponding reaction rates. The conservation equations for the different species can then be written as

$$
\begin{aligned}
& \frac{\partial c_{1+}}{\partial t}+\frac{\partial \mathcal{F}_{1+}}{\partial x}=-Q_{1}-Q_{2}, \\
& \frac{\partial c_{1-}}{\partial t}+\frac{\partial \mathcal{F}_{1-}}{\partial x}=-Q_{1}-Q_{3}, \\
& \frac{\partial c_{20}}{\partial t}+\frac{\partial \mathcal{F}_{20}}{\partial x}=Q_{1}-Q_{2}-Q_{3}, \\
& \frac{\partial c_{3+}}{\partial t}+\frac{\partial \mathcal{F}_{3+}}{\partial x}=Q_{2}, \\
& \frac{\partial c_{3-}}{\partial t}+\frac{\partial \mathcal{F}_{3-}}{\partial x}=Q_{3},
\end{aligned}
$$

where $F_{(\cdot)}$ are the fluxes of the respective species. Reactions at the electrodes can now be written as

$$
\begin{aligned}
(I) & L i & \rightleftharpoons L i^{+}+e^{-}, \\
(I I) & L i+N^{-} & \rightleftharpoons L i N+e^{-}, \\
(I I I) & L i+L i N & \rightleftharpoons L i_{2} N^{+}+e^{-} .
\end{aligned}
$$

At the anode the boundary conditions pertaining to the flux of each species can now be stated as, cf. (4a)-(4c),

$$
\begin{aligned}
\left.\mathcal{F}_{1+}\right|_{x=0} & =r_{(I)}, \\
\left.\mathcal{F}_{1-}\right|_{x=0} & =-r_{(I I)}, \\
\left.\mathcal{F}_{20}\right|_{x=0} & =-r_{(I I I)}+r_{(I I)}, \\
\left.\mathcal{F}_{3+}\right|_{x=0} & =r_{(I I I)}, \\
\left.\mathcal{F}_{3-}\right|_{x=0} & =r_{(I)} .
\end{aligned}
$$

Now let us define $\mathcal{F}_{T}$ as, cf. (9),

$$
\mathcal{F}_{T}=\mathcal{F}_{1+}+\mathcal{F}_{1-}+2 \mathcal{F}_{20}+3 \mathcal{F}_{3+}+3 \mathcal{F}_{3-},
$$

which at the anode gives, cf. (11),

$$
\left.\mathcal{F}_{T}\right|_{x=0}=r_{(I)}+r_{(I I)}+r_{(I I I)}=\frac{j}{F}
$$

and similarly for the cathode we can write,

$$
\left.\mathcal{F}_{T}\right|_{x=L}=\frac{j}{F} .
$$

If we also assume, cf. (9),

$$
c_{T}=c_{1+}+c_{1-}+2 c_{20}+3 c_{3+}+3 c_{3-},
$$


then we can write, cf. (10),

$$
\frac{\partial c_{T}}{\partial t}+\frac{\partial \mathcal{F}_{T}}{\partial x}=0
$$

Thus, starting from equations (48) and (49) and following the approach laid down in Section 4 , we can show that the transport equation for this extended system will again be formally equivalent to the Plank-Nernst equation (1). This analysis can be further extended in the same way to fourth- and higher-order ion clusters which will lead to the same conclusions.

\section{References}

[1] J. Newman and K. E. Thomas-Alyea, Electrochemical Systems. John Wiley and Sons, 2004.

[2] A. K. Sethurajan, S. A. Krachkovskiy, I. C. Halalay, G. R. Goward, and B. Protas, "Accurate Characterization of Ion Transport Properties in Binary Symmetric Electrolytes Using In Situ NMR Imaging and Inverse Modeling," The Journal of Physical Chemistry $B$, vol. 119, no. 37, pp. 12238-12248, 2015.

[3] S. A. Krachkovskiy, J. D. Bazak, S. Fraser, , I. C. Halalay, and G. R. Goward, "Determination of Mass Transfer Parameters and Ionic Association of $\mathrm{LiPF}_{6}$ : Organic Carbonates Solutions," J. Electrochem. Soc., vol. 164, no. 4, pp. A912-A916, 2017.

[4] S. Zugmann, M. Fleischmann, M. Amereller, R. M. Gschwind, H. D. Wiemhfer, and H. J. Gores, "Measurement of Transference Numbers for Lithium Ion Electrolytes via Four Different Methods, a Comparative Study," Electrochim. Acta, vol. 56, no. 11, pp. 3926-3933, 2011.

[5] M. Gouverneur, F. Schmidt, and M. Schönhoff, "'Negative Effective Li Transference Numbers in Li Salt/Ionic Liquid Mixtures: Does Li Drift in the "Wrong" Direction?"," Phys. Chem. Chem. Phys., vol. 20, pp. 7470-7478, 2018.

[6] L. Onsager, "Deviations from Ohm's Law in Weak Electrolytes," The Journal of Chemical Physics, vol. 2, no. 9, pp. 599-615, 1934.

[7] L. Onsager, "Initial Recombination of Ions," Phys. Rev., vol. 54, pp. 554-557, Oct 1938.

[8] H. Dai and T. A. Zawodzinski, "The Dependence of Lithium Transference Numbers on Temperature, Salt Concentration and Anion Type in Poly (Vinylidene Fluoride)-Hexafluoropropylene Copolymer-Based Gel Electrolytes," J. Electroanal. Chem., vol. 459, no. 1, pp. 111-119, 1998.

[9] T. D. Claridge, High-Resolution NMR Techniques in Organic Chemistry, vol. 27. Newnes, 2008.

[10] A. Nyman, M. Behm, and G. Lindbergh, "Electrochemical Characterisation and Modelling of the Mass Transport Phenomena in LiPF $:$ EMC Electrolyte," Electrochim. Acta, vol. 53, no. 22, pp. 6356-6365, 2008. 
[11] J. Liu and C. W. Monroe, "Solute-Volume Effects in Electrolyte Transport," Electrochimica Acta, vol. 135, pp. 447-460, 2014.

[12] M. M. Doeff, P. Georèn, J. Qiao, J. Kerr, and L. De Jonghe, "Transport Properties of a High Molecular Weight Poly (propylene oxide)- $\mathrm{LiCF}_{3} \mathrm{SO}_{3}$ System," Journal of the Electrochemical Society, vol. 146, no. 6, pp. 2024-2028, 1999.

[13] R. A. Robinson and R. H. Stokes, Electrolyte Solutions. Butterworth \& Co. Ltd., second ed., 1970.

[14] M. Doyle, T. F. Fuller, and J. Newman, "The Importance of the Lithium Ion Transference Number in Lithium/Polymer Cells," Electrochimica Acta, vol. 39, no. 13, pp. 20732081, 1994.

[15] C. W. Monroe and C. Delacourt, "'Continuum Transport Laws for Locally Non-Neutral Concentrated Electrolytes"," Electrochimica Acta, vol. 114, pp. 649 - 657, 2013.

[16] C. M. Tenney and R. T. Cygan, "Analysis of Molecular Clusters in Simulations of Lithium-Ion Battery Electrolytes," The Journal of Physical Chemistry C, vol. 117, no. 47, pp. 24673-24684, 2013.

[17] Y. Marcus and G. Hefter, "Ion Pairing," Chemical Reviews, vol. 106, no. 11, pp. 45854621, 2006.

[18] V. Srinivasan and J. Newman, "Design and Optimization of a Natural Graphite/Iron Phosphate Lithium-Ion Cell," Journal of the Electrochemical Society, vol. 151, no. 10, pp. A1530-A1538, 2004.

[19] O. Borodin and G. D. Smith, "Quantum Chemistry and Molecular Dynamics Simulation Study of Dimethyl Carbonate: Ethylene Carbonate Electrolytes Doped with LiPF 6 ," The Journal of Physical Chemistry B, vol. 113, no. 6, pp. 1763-1776, 2009.

[20] A. Dukhin and S. Parlia, "Ion-Pair Conductivity Theory Fitting Measured Data for Various Alcohol-Toluene Mixtures across Entire Concentration Range," J. Electrochem. Soc., vol. 162, no. 4, pp. H256-H263, 2015.

[21] M. Klett, M. Giesecke, A. Nyman, F. Hallberg, R. W. Lindström, G. Lindbergh, and I. Furo, "Quantifying Mass Transport during Polarization in a Li Ion Battery Electrolyte by in Situ Li NMR Imaging," J. Am. Chem. Soc., vol. 134, no. 36, pp. 14654-14657, 2012.

[22] V. Bukshtynov, O. Volkov, and B. Protas, "On Optimal Reconstruction of Constitutive Relations," Physica D: Nonlinear Phenomena, vol. 240, no. 16, pp. 1228-1244, 2011.

[23] V. Bukshtynov and B. Protas, "Optimal Reconstruction of Material Properties in Complex Multiphysics Phenomena," J. Comput. Phys., vol. 242, pp. 889-914, 2013. 
[24] J. Vatamanu, O. Borodin, and G. D. Smith, "Molecular Dynamics Simulation Studies of the Structure of a Mixed Carbonate $\mathrm{LiPF}_{6}$ Electrolyte Near Graphite Surface as a Function of Electrode Potential," The Journal of Physical Chemistry C, vol. 116, no. 1, pp. 1114-1121, 2011. 
Table 1: Typical values of dimensional parameters.

\begin{tabular}{lllll}
\hline Parameter & Symbol & Value & Units & Source \\
\hline Debye Length & $L_{D}$ & $O\left(10^{-9}\right)$ & $m$ & {$[24]$} \\
Cell Length & $L$ & $O\left(10^{-2}\right)$ & $m$ & Experiment \\
Reaction Rate & $k$ & $O\left(10^{9}\right)$ & $\frac{1}{s}$ & {$[19]$} \\
Time of Experiment & $\tau$ & $O\left(10^{4}\right)$ & $s$ & Experiment \\
Concentration & $\hat{c}$ & $10^{3}$ & $\frac{m o l}{m^{3}}$ & Experiment \\
\hline
\end{tabular}




\section{List of Figures}

1 Concentration profiles obtained via in-situ magnetic resonance imaging during a galvanostatic polarization experiment. . . . . . . . . . . . . . .

2 Constant and concentration-dependent effective salt diffusivities (left) and $\mathrm{Li}^{+}$transference numbers (right) reconstructed from the measurement data described in Section 2 using the inverse modelling approach (problems P1 and P2) defined in Appendix A) based on system (1). . . . . . . . . . . . . . . 


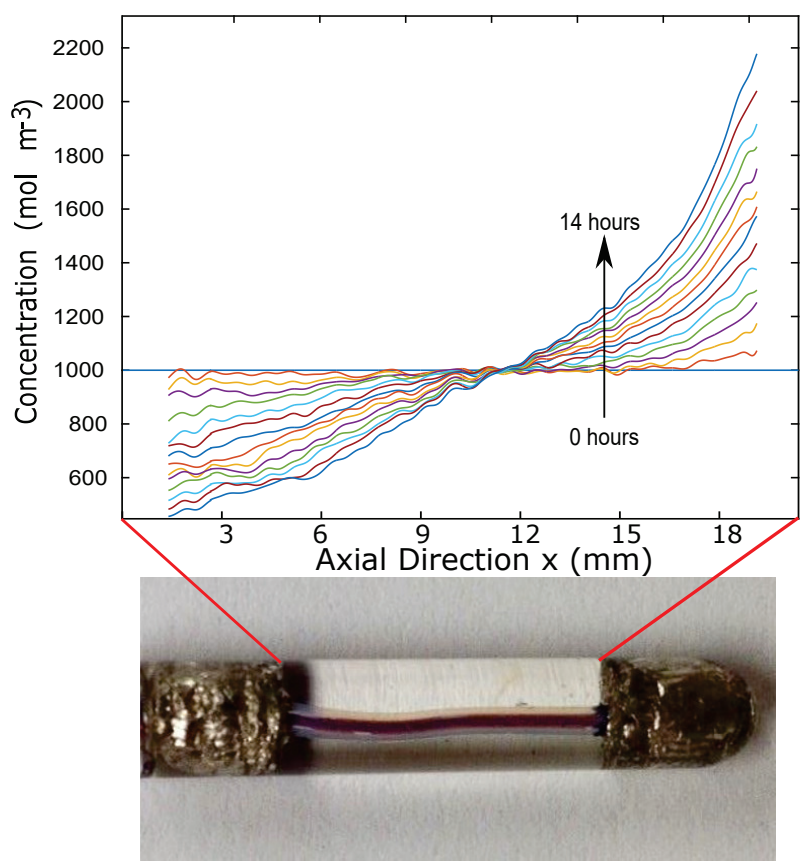

Figure 1: Concentration profiles obtained via in-situ magnetic resonance imaging during a galvanostatic polarization experiment. 

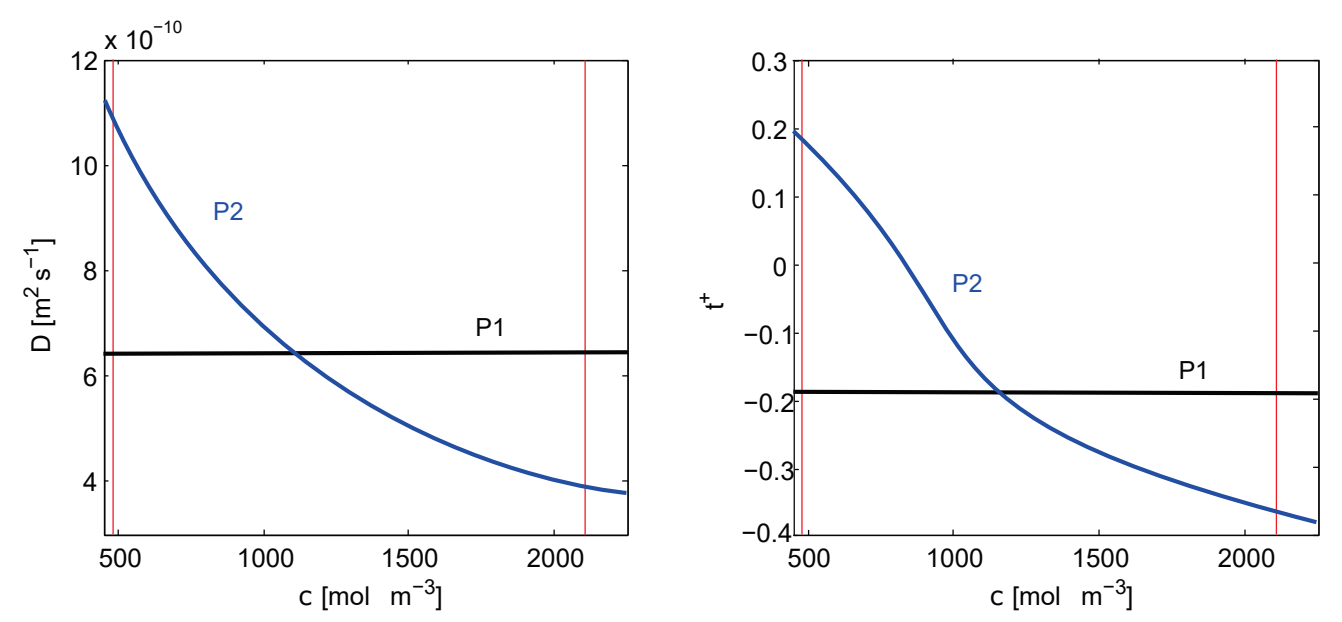

Figure 2: Constant and concentration-dependent effective salt diffusivities (left) and $\mathrm{Li}^{+}$ transference numbers (right) reconstructed from the measurement data described in Section 2 using the inverse modelling approach (problems P1 and P2) defined in Appendix A) based on system (1). 